\section{The Absolute Power of Sports Medicine}

\section{Jane P. Sheldon}

1 t happened in the first quartile of the game, when there was a sudden distribution of falling bodies-a local behavior that occurs with relatively high frequency in college basketball. At that moment, star center Newman Keuls's jump discontinuity caused him to experience some painful joint variation on the left-hand side. The instant replay gave direct proof that the floor function had caused his left leg's rotation and skewness. Keuls's fractional expression was a radical sign that he was in severe pain. Significant swelling and bruising of his knee contributed to the effect size, so there was no denying the consequent.

The team doctor made the logical proposition that Keuls's leg would need to be union set in a linear association. However, he also quickly discerned that the disjoint events occurring in Keuls's knee were not the only injuries. On Keuls's torso the doctor could also see an inscribed circle in the area below curves. The locus of this independent injury was next to the latus rectum, which is, needless to say, a critical point. In this lateral surface area there was a hole leading to the inner product. The locus and partitions of this open wound shocked the physician, for he was able to see numerous exposed chords, scalars, fractals, dot products, and other odd functions. Due to these critical values, he rushed Keuls to the emergency room using optimal transport.

The emergency room staff adhered to l'Hôpital's rule, which necessitated that Keuls's injuries received immediate, standard action. First, the nurses used the washer method to eliminate all residuals within the wound. Then, the surgeons followed this discrete procedure with exhaustive linear operations (the graphic methods of which I'll spare you). Fortunately, the operations on his knee and open wound were without chance errors, and the doctors have total confidence in the outcomes. In fact, the expected value of these treatments is that they will result in a normal approximation, with no limits, of Keuls's preinjury parameters.

After several months of rehabilitation, Keuls's attitude is excellent. According to the medical experts, there is a high probability that he will recover quickly, gain full function, once again don his uniform, and soon reach his prime.

University of Michigan-Dearborn

4901 Evergreen Rd.

Dearborn, Ml 48128

USA

e-mail: jsheldon@umich.edu 